\title{
Partial Immobilization and Non-Instantaneous Equilibrium Models for Gas Membrane Transport Mechanism
}

\author{
Haoyu Wu, Boguslaw Kruczek, Jules Thibault \\ Department of Chemical and Biological Engineering, University of Ottawa \\ 161 Louis Pasteur St., Ottawa, Canada K1N 6N5 \\ hwu055@uottawa.ca; bkruczek@uottawa.ca; jules.thibault@uottawa.ca
}

\section{Extended Abstract}

Solution-diffusion and dual-mode sorption models are commonly used as transportation models for the characterization of the gas permeation through a polymer film [1-3]. The solution-diffusion model depicts mathematically two processes of a gas permeating through a rubbery film: gas sorption and desorption processes happening at the two interfaces of the film; and gas diffusion process within the film [4,5].They are represented by Henry's law and Fick's second law of diffusion (Eq.(1)), respectively.

$$
\frac{\partial C}{\partial t}=D \frac{\partial^{2} C}{\partial x^{2}}
$$

In Equation (1), $C$ is the concentration in Henry's sites (the only available sites), $D$ is the diffusivity of gas molecules within the membrane, $t$ is the transport time and $x$ is the transport distance. Dual-mode sorption augments the solutiondiffusion model with a Langmuir adsorption process, which is a process where gas molecules occupy so called "Langmuir's sites", which can be described as relaxed volumes resulting from glassy polymer chain movement during the diffusion process $[1,3,6,7]$. In addition to the characteristic diffusion process through the membrane, there is a gas molecule exchange between Henry's and Langmuir's sites to establish equilibrium concentration between gas molecule populations [8]. The extension of the solution-diffusion model made it possible to describe mathematically the nonlinear behaviour of gas transport through a glassy polymer. However, the dual-mode sorption model is in some cases a simplification of the underlying process due to two main assumptions: (1) equilibrium between Henry's and Langmuir's sites is established instantaneously, and (2) gas molecules trapped into the Langmuir's sites are completely immobile [1,3,6-9]. Although these simplifications made the problem-solving easier, they sometimes over-approximate the complexity of the actual gas transportation behaviour.

Partial immobilization model and non-instantaneous equilibrium model are complex transportation models established based on the solution-diffusion and dual-mode sorption models $[1,3,8,9]$. In this paper, a model combining the two models is considered where two key parameters are added into the dual-mode sorption model: (1) a partial immobilization factor $(F)$ in Eq. (2)

$$
C_{m}=C_{D}+F C_{H}
$$

where $C_{m}$, the concentration participating in the diffusion process, is an addition of gas concentration in Henry's sites $\left(C_{D}\right)$ and the concentration of the mobile Langmuir sites $\left(\mathrm{F} C_{H}\right)$ and $F$, the partial immobilization factor, is the ratio of the amount of mobile gas molecules in Langmuir's sites having the same diffusivity as the gas molecules in Henry's sites and the amount of immobile gas molecules in Langmuir's sites; (2) a non-instantaneous equilibrium factor $\left(k_{a d}\right)$ in Eq. (2). The non-instantaneous equilibrium factor $\left(k_{a d}\right)$ is the adsorption rate constant. Together with the hole affinity constant $b$ in dualmode sorption, it represents the rate at which the equilibrium between Henry's and Langmuir's site concentrations is established. The new model is represented by transportation Equations (3) and (4). 


$$
\begin{gathered}
\frac{\partial C_{D}}{\partial t}=D \frac{\partial^{2} C_{D}}{\partial x^{2}}-\dot{m}_{D \rightarrow H}\left(k_{a d}, b\right) \\
\frac{\partial C_{H}}{\partial t}=F D \frac{\partial^{2} C_{H}}{\partial x^{2}}+\dot{m}_{D \rightarrow H}\left(k_{a d}, b\right)
\end{gathered}
$$

Where $\dot{m}_{D \rightarrow H}\left(k_{a d}, b\right)$ is the gas molecule transfer rate between Henry's sites and Langmuir's sites and the value is related to $k_{a d}$ and $b$. The new model is a better representation of some actual gas permeation process through a glassy polymer film $[10,11]$. Currently, there is no analytical solution for this more complex model such that it needs to be solved numerically.

In the paper, the discussion on the new model will be focussed on seven special combinations of $F$ and $k_{a d}$. The simulated upstream and downstream time lags are presented in Figure 1. The seven combinations are: (1) $k_{a d}=0, F=0$ : Linear model (solution-diffusion model) (1), (2), (3) in Figure 1); (2) $k_{a d}=\infty, F=0$ : Instantaneous equilibrium and complete immobilization model (Dual-mode sorption model) (7) in Figure 1); (3) $k_{a d}=\infty, 0<F<1$ : Instantaneous equilibrium and partial immobilization model (6) in Figure 1); (4) $k_{a d}=\infty, F=1$ : Instantaneous equilibrium and complete mobilization model (5) in Figure 1); (5) $k_{a d}>0, F=0$ : Non-instantaneous equilibrium and complete immobilization model (8) in Figure 1); (6) $k_{a d}>0,0<F<1$ : Non-instantaneous equilibrium and partial immobilization model (9) in Figure 1); (7) $k_{a d}>0, F=1$ : Non-instantaneous equilibrium and complete mobilization model (4) in Figure 1). The specific features of these cases will be discussed in details based on their permeation mechanisms, pressure accumulation, and both the upstream and downstream dynamic time lag curves.

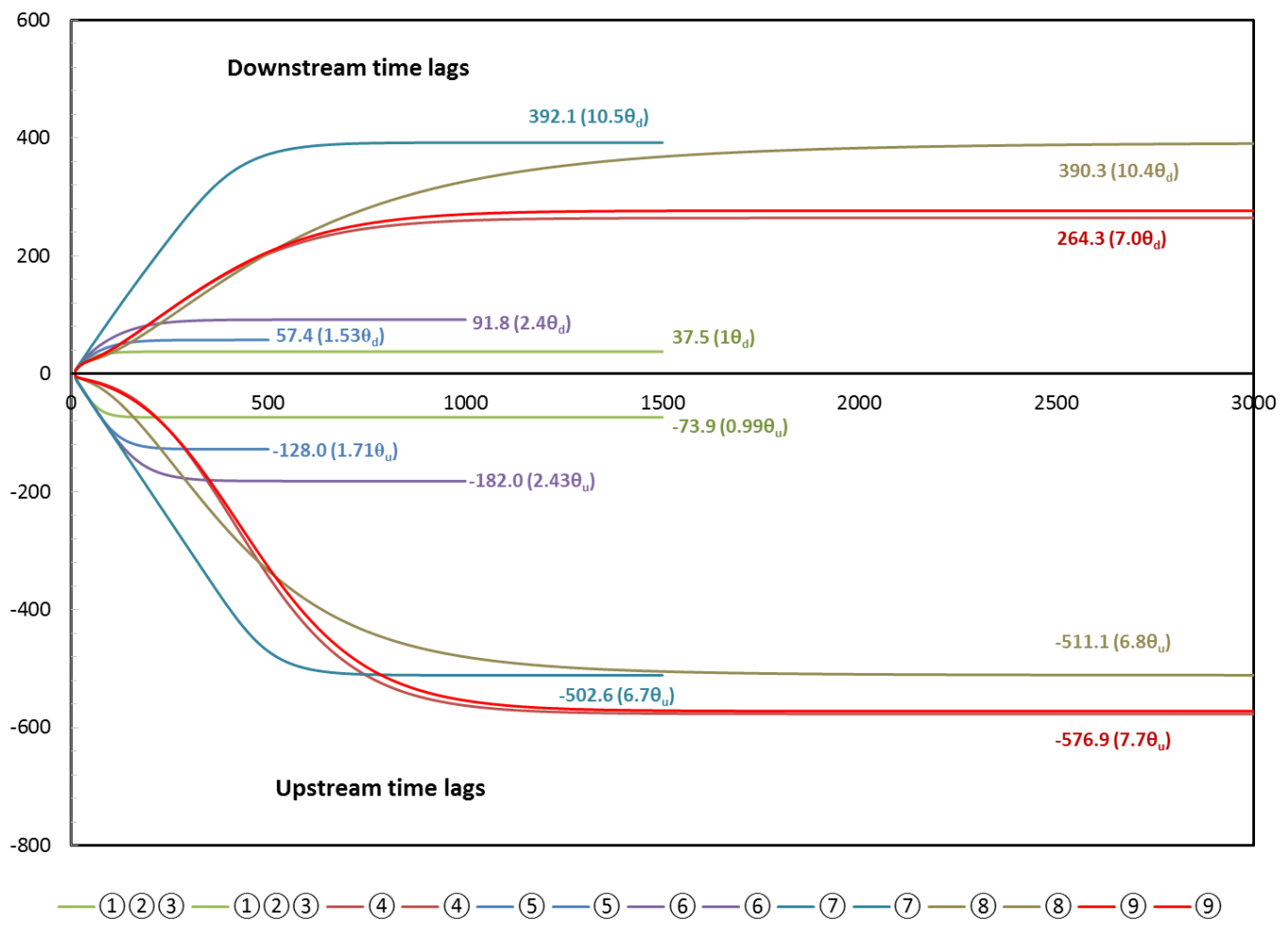

Fig. 1: Simulation results of upstream and downstream time lags under the feed pressure of 2 atm with the following membrane properties: Diffusivity $\left(4 \times 10^{-12} \mathrm{~m}^{2} / \mathrm{s}\right)$, Solubility $\left(7.44 \mathrm{~mol} / \mathrm{m}^{3} \mathrm{~Pa}\right)$, Hole saturation constant $C_{H}{ }^{\prime}\left(67.4 \mathrm{~mol} / \mathrm{m}^{3}\right)$, Hole affinity constant $b\left(6 \times 10^{-2} \mathrm{~Pa}^{-1}\right)$. (1) $\mathrm{F}=0, \mathrm{k}_{\mathrm{ad}}=0$; (2) $\mathrm{F}=0.5, \mathrm{k}_{\mathrm{ad}}=0$; (3) $\mathrm{F}=1, \mathrm{k}_{\mathrm{ad}}=0$; (4) $\mathrm{F}=1, \mathrm{k}_{\mathrm{ad}}=0.001$; (5) $\mathrm{F}=1, \mathrm{k}_{\mathrm{ad}}=\infty$; (6) $\mathrm{F}=0.5, \mathrm{k}_{\mathrm{ad}}=$ $\infty$; (7) $\mathrm{F}=0, \mathrm{k}_{\mathrm{ad}}=\infty ;(8) \mathrm{F}=0, \mathrm{k}_{\mathrm{ad}}=0.001 ;$ (9) $\mathrm{F}=0.5, \mathrm{k}_{\mathrm{ad}}=0.001$ ). 


\section{References}

[1] L. Wang, J.-P. Corriou, C. Castel, and E. Favre, "Transport of Gases in Glassy Polymers under Transient Conditions: Limit-Behavior Investigations of Dual-Mode Sorption Theory," Industrial \& Engineering Chemistry Research, vol. 52, no. 3, pp. 1089-1101, 2013.

[2] D. R. Paul, "Gas Sorption and Transport in Glassy Polymers," Berichte der Bunsengesellschaft für physikalische Chemie, vol. 83, no. 4, pp. 294-302, 1979.

[3] D. R. Paul and W. J. Koros, "Effect of partially immobilizing sorption on permeability and the diffusion time lag," Journal of Polymer Science: Polymer Physics Edition, vol. 14, no. 4, pp. 675-685, 1976.

[4] R. M. Barrer, J. A. Barrie, and J. Slater, "Sorption and diffusion in ethyl cellulose. Part III. Comparison between ethyl cellulose and rubber," Journal of Polymer Science, vol. 27, no. 115, pp. 177-197, 1958.

[5] J. G. Wijmans and R. W. Baker, "The solution-diffusion model: a review," Journal of Membrane Science, vol. 107, no. 1-2, pp. 1-21, 1995.

[6] H. Odani and T. Uyeda, "Theories of Sorption and Transport in Polymer Membrane," Polymer Journal, vol. 23, no. 5, pp. 467-479, 1991.

[7] N. Al-Qasas, J. Thibault, and B. Kruczek, "The effect of the downstream pressure accumulation on the time-lag accuracy for membranes with non-linear isotherms," Journal of Membrane Science, vol. 511, pp. 119-129, 2016.

[8] J. A. Tshudy and C. V. Frankenberg, "A model incorporating reversible immobilization for sorption and diffusion in glassy polymers,” Journal of Polymer Science: Polymer Physics Edition, vol. 11, no. 10, pp. 2027-2037, 1973.

[9] J. Guo and T. A. Barbari, "A Dual Mode, Local Equilibrium Relaxation Model for Small Molecule Diffusion in a Glassy Polymer," Macromolecules, vol. 41, no. 1, pp. 238-245, 2008.

[10] S. K. Burgess, O. Karvan, J. Johnson, R. M. Kriegel, and W. J. Koros, "Oxygen sorption and transport in amorphous poly(ethylene furanoate)," Polymer, vol. 55, no. 18, pp. 4748-4756, 2014.

[11] J. T. Vaughn, W. J. Koros, J. Johnson, and O. Karvan, "Effect of thermal annealing on a novel polyamide-imide polymer membrane for aggressive acid gas separations," Journal of Membrane Science, vol. 401-402, pp. 163-174, 2012. 